\title{
Feasibility Study of the Unmanned-aerial-vehicle Radio-frequency Identification System for Localizing Construction Materials on Large-scale Open Sites
}

\author{
Sanghyeok Kang, ${ }^{1}$ Man-Woo Park, ${ }^{2 *}$ and Wonho Suh $^{3}$ \\ ${ }^{1}$ Department of Civil \& Environmental Engineering, Incheon National University, \\ Yeonsu-gu, Incheon 22012, South Korea \\ ${ }^{2}$ Department of Civil \& Environmental Engineering, Myongji University, \\ Cheoin-gu, Yongin 17058, South Korea \\ ${ }^{3}$ Department of Transportation \& Logistics Engineering, Hanyang University, \\ Sangnok-gu, Ansan 15588, South Korea
}

(Received April 18, 2018; accepted March 13, 2019)

Keywords: unmanned aerial vehicle, radio frequency identification, material management, tracking

The locations of construction resources, such as workers, equipment, and materials, are one of the important data types for monitoring the states of ongoing activities. The needs for location data have driven various research efforts on sensing technologies for tracking these resources. Although a GPS has been employed mostly for construction equipment, radio frequency identification (RFID) technology has been used for construction materials including precast elements. The RFID system generally requires an on-site manager to walk around the site holding a reader to communicate with the tags attached to materials at close ranges. These manual procedures are time-consuming and inefficient when the materials are spread over a large-scale broad area. Also, the materials located beyond a manager's reach are undetectable. Therefore, in this study, an unmanned aerial vehicle (UAV)-based system that carries an RFID reader in the air for locating RFID tags attached to materials on the ground is proposed. The proposed UAV-RFID system receives signals from the tags located within the sensing range of the reader and saves their strength indicator values. The candidate area of the tag locations is estimated from the specifications of the reader and the flight altitude. Experimental tests signify that the system has a great potential to replace the manual reading process and improve the material management efficiency.

\section{Introduction}

It is very important to identify the location of materials in a construction site to improve job site productivity in terms of time shortening and cost reduction. In a construction project, which usually has a high proportion of direct costs, the well-organized management of construction materials directly affects the overall construction cost. Construction sites are usually filled with many types of materials to be fabricated on the site. Failure to identify 
the material location leads to increases in cost and time because specific materials cannot be promptly retrieved. Therefore, cost and time management in a project cannot be performed properly without acquiring the accurate location of materials.

Recently, interest in modular construction has been growing rapidly because the modular construction processes can improve construction efficiency markedly by manufacturing structural elements at a factory in advance to be assembled on the site. In modular construction processes, premanufactured modules should be managed carefully from their delivery to installation. Considering modules as a type of material, material management becomes more critical as it directly affects the overall efficiency of the construction project. For example, in a large-scale plant construction project, a large number of materials such as H-beams and steel pipes are transported from a factory and piled over the site, and it often takes a lot of time to retrieve certain materials because the location of the materials is not appropriately identified. In the case of building construction, various types of interior and exterior materials are transported to the site in advance for their fabrication. If the location of the materials is not promptly available, the procurement of the materials may be delayed accordingly. Material management becomes much more important in the projects of curtain wall construction since the construction involves hundreds of elements, each of which has its own fabrication sequence and position. On-site materials should also be managed appropriately to avoid theft or embezzlement that occurs occasionally on the site. Recently, the government of South Korea has supplemented the policy on construction materials after continuous incidents of rock bolt thefts on the construction sites of highway tunnel projects. The policy supplement signifies and emphasizes the importance of material management.

In most construction sites, the locations of most materials are generally identified through manual processes. A few types of materials with higher impact on the project may be managed using radio frequency identification (RFID) technology. On-site RFID systems can be divided into two types. The first type is installing an RFID reader at a fixed position to collect data in the presence of equipment or materials in a certain range around the fixed position. However, this approach has a limitation in that the presence of equipment and materials can be identified within very limited areas. The other type relies on an on-site worker who patrols the site, while carrying an RFID reader to collect location data of materials; however, this approach is timeconsuming and error-prone. In particular, it takes a lot of time in a large-scale site and the data collected are often inaccurate. It is also difficult to obtain data of the materials stored in places that are difficult for the worker to access because the RFID system has a limited range of communication. These inefficient processes may lead to schedule delays and increased costs.

To solve the problems stated above, a material location tracking system using unmanned aerial vehicles (UAVs) and an RFID system is proposed in this study. In short, the UAVs are employed to make a typical RFID system mobile and provide the reference location of the tag via the GPS unit installed on the UAVs. In this paper, we present the integration of a UAV and an RFID system in terms of the hardware setup and the required data processing for localization. The important factors to consider in the localization are discussed and a series of experiments are presented in detail indicating the procedures used to determine important factors and validate the feasibility of material localization. 


\section{Background}

RFID technology has been widely used in various areas such as retail, logistics, supply chain management, and security. In the construction sector, many attempts have been made to utilize RFID for construction management, and RFID technology has been proved to have great potential in improving construction project management goals in terms of time, quality, cost, safety, and environment. ${ }^{(1)}$ Jaselskis et al. ${ }^{(2)}$ presented RFID applications in the construction industry-concrete operations, human resource and equipment allocations, and material controland concluded that RFID technology could appropriately manage and track important materials. Akinci et $a l^{(3)}$ discussed the utilization of RFID for tracking precast concrete components and their historical information from fabrication to postconstruction and proposed the utilization of RFID technology from a large-scale precast manufacturer/erector's perspective. Jaselskis and El-Misalami showed that RFID tags reduced the time required to download data into a company's material tracking system. ${ }^{(4)}$ They demonstrated that RFID is a promising technology for on-site material management tasks. Goodrum et al. developed a tracking and inventory system that is capable of storing operation and maintenance (O\&M) data using active RFID tags. ${ }^{(5)}$ The literature presented that active RFID systems can be used to store pertinent O\&M data on inventory tools in construction environments despite metal interference and low temperatures.

Ergen et al. proposed an automated system using RFID technology combined with a GPS. ${ }^{(6)}$ This prototype system was successfully tested in the field at a precast storage yard. Yin et al. developed a precast production management system for the inspection of incoming materials, production process, molds, specimen strength feed, and logistic and receiving management using personal digital assistants (PDAs) and RFID. ${ }^{(7)}$ Lu et al. investigated how RFID can be used in the management of materials, men, and machinery for construction projects, and proposed various scenarios that can illustrate the uses of RFID technology in construction project management. ${ }^{(1)}$ Valero and Adan demonstrated that the integration of RFID technology with other systems, such as RFID + vision systems, RFID + positioning systems, and RFID + microcomputers, can make the whole control system more accurate and efficient as well as enhance the feasibility and applications of RFID in construction. ${ }^{(8)}$

UAV systems are becoming attractive for many applications in the construction sector. UAVs are usually used for collecting aerial photograph data over a construction site taking advantage of its mobility in air. The image data obtained by UAVs are used for various purposes such as inspecting and monitoring infrastructure facilities and safety management. Rathinam et al. presented a detection algorithm that can identify and localize various structures including highways and canals. ${ }^{(9)}$ Zhang and Elaksher introduced a UAV-based digital imaging system for collecting surface condition data over rural roads. ${ }^{(10)}$ Their experiments showed that the system is capable of providing three-dimensional (3D) surface information for road condition assessment. Irizarry et al. applied drone technology for safety management in a construction site. $^{(11)}$ They counted hardhats viewed through the display of a mobile device on the site to evaluate the performance of the drone application and concluded that using the drone together with a large interface would be as accurate as having a safety manager with a plain view of the jobsite. Siebert and Teizer evaluated a UAV system built to rapidly and autonomously acquire 
mobile 3D mapping data. ${ }^{(12)}$ They concluded that the UAV application of surveying earthworks is useful to researchers and practitioners.

Ashour et al. developed a real-time drone system for facility inspection and violation detection in construction sites. ${ }^{(13)}$ Similarly, Metni and Hamel developed a novel control law that uses the homography matrix computed from the information obtained from the UAV vision system and applied it to bridge maintenance. ${ }^{(14)}$ Morgenthal and Hallermann examined the use of UAVs for visual inspection and damage detection on civil structures and concluded that damage assessment quality may be quantified. ${ }^{(15)}$ Bang et al. proposed a method of generating a panorama of a large construction site using UAVs, which helps managers easily identify construction site conditions. ${ }^{(16)}$

Various state-of-the-art technologies are being combined to resolve problems in a construction site. RFID technology can create a synergistic effect with UAVs. As previously implied, the integration with UAVs enables capturing data in a more rapid and accurate way. RFID applications can automate the collection of information about the location of materials. This benefit provides cost reduction with greater accuracy and reliability than is possible with manual methods. UAVs can provide mobility as they can fly into zones that are dangerous to human life. Therefore, in this paper, we combine the two technologies to enhance the practices of material management on a construction site.

\section{Problem Statement and Objective}

Construction sites generally involve various types of materials being delivered, stored, and installed throughout construction periods. As a critical type of on-site resource to manage, materials should be carefully monitored on construction sites in terms of their locations as well as quality. When the number of material types involved in a construction project is large, and the construction site is very crowded or large, it becomes relatively difficult to monitor the material locations. For the effective monitoring of the material locations, RFID technologies have been applied in many projects. Currently, there are two types of RFID readers-hand-held and fixed readers. However, the current practices of the RFID-based material localization have several limitations to overcome. The use of hand-held readers requires on-site workers to patrol a site to read all the tags attached to materials. This process is time-consuming especially when materials are spread over a large-scale site. The use of fixed readers can retrieve location data only when materials pass near the readers. In other words, the former and latter have limitations in terms of time cost and sensing coverage, respectively. In this study, we aim to overcome the limitations (Fig. 1) by proposing a new platform that integrates UAVs and RFID. The proposed platform localizes tags attached to materials based on the tag ID data collected by the RFID reader installed on a UAV as well as the GPS location data of the UAV. The proposed platform has a potential to resolve the limitation of hand-held readers by replacing humans with UAVs that can fly over a construction site within a short time and access the spaces that are difficult for workers to reach. The mobility of a UAV can also be a key to resolve the limitation of fixed readers. Moreover, we investigate the specifications of RFID readers and tags to study the feasibility of the integration of UAVs and RFID for on-site localization purposes. In 


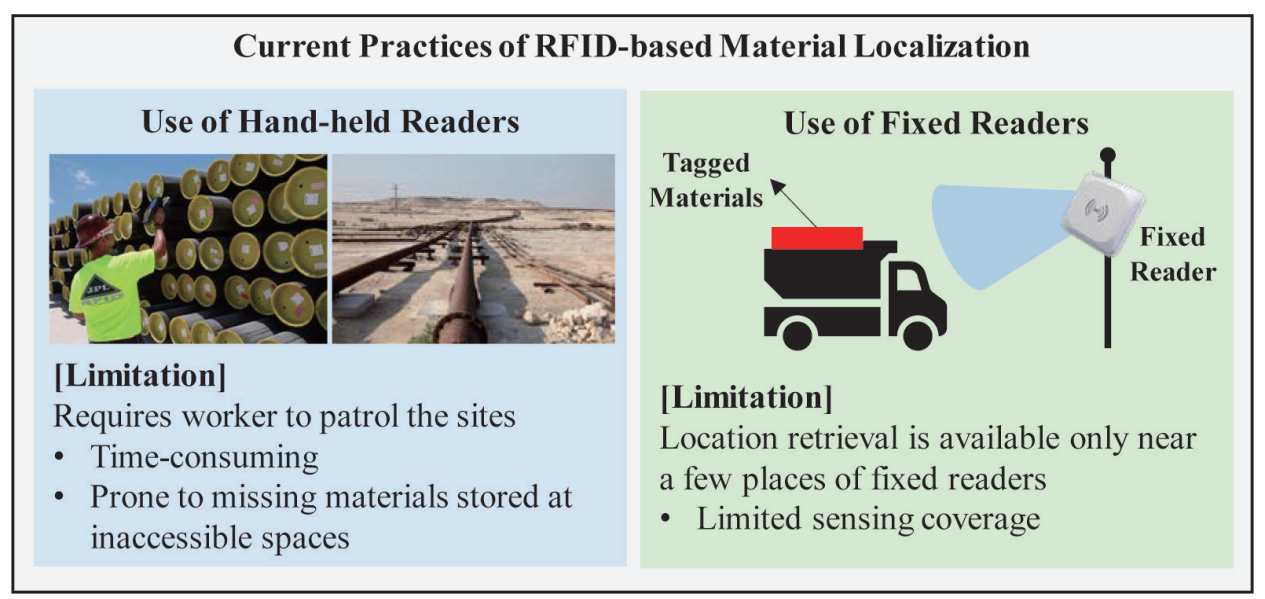

Fig. 1. (Color online) Current practices of RFID-based material localization.

this paper, we present various experiments that determine the sensitivity of the RFID tags and readers as well as the factors that affect the tag reading performance. Finally, the feasibility of the proposed platform is validated for localizing materials spread over a flat ground. Note that the objective of this paper is limited to the feasibility test signifying the potential of the UAV-RFID platform, and more accurate localization for practical applications of the platform is beyond the scope of this study and remains as a future work. Also, in the experiments, metal materials are focused and all materials are spread on the ground.

\section{4. $\quad$ Proposed Methods}

Figure 2 presents the basic concept and overall framework of the proposed platform. It basically uses a UAV that is equipped with a GPS unit and an RFID reader to collect UAV locations and RFID tag IDs. The RFID tag IDs are collected with received signal strength indicator (RSSI) data and time-synchronized with GPS data. When a tag is identified, then its location can be registered as the UAV location that is the time-synchronized GPS data. Since the UAV location is located at a certain level of altitude and the RFID reader reads all tags located within a certain range around the reader, the tag locations are not the same as the UAV location. Further in-depth data analysis may require pin-pointing the tag locations using the RSSI data, which is generally inversely proportional to the distance between the reader and a tag. However, the approximate localization that discovers a certain range where a tag is located, relying only on the GPS data, can still be a competitive alternative to current practices. Therefore, in this paper, we focus on the use of the GPS and RFID tag reading and leave the analysis of RSSI data for precise localization as a future work.

In other words, in this study, we mainly test the feasibility of the combination of UAVs and RFID for reading RFID tags attached to on-site materials through a flight of more than $10 \mathrm{~m}$ height. The hardware and software employed for data collection will be elaborated, and a series of experiments will be presented in detail to discover the specifications of the employed hardware, to investigate the appropriate settings for material localization, and to validate 


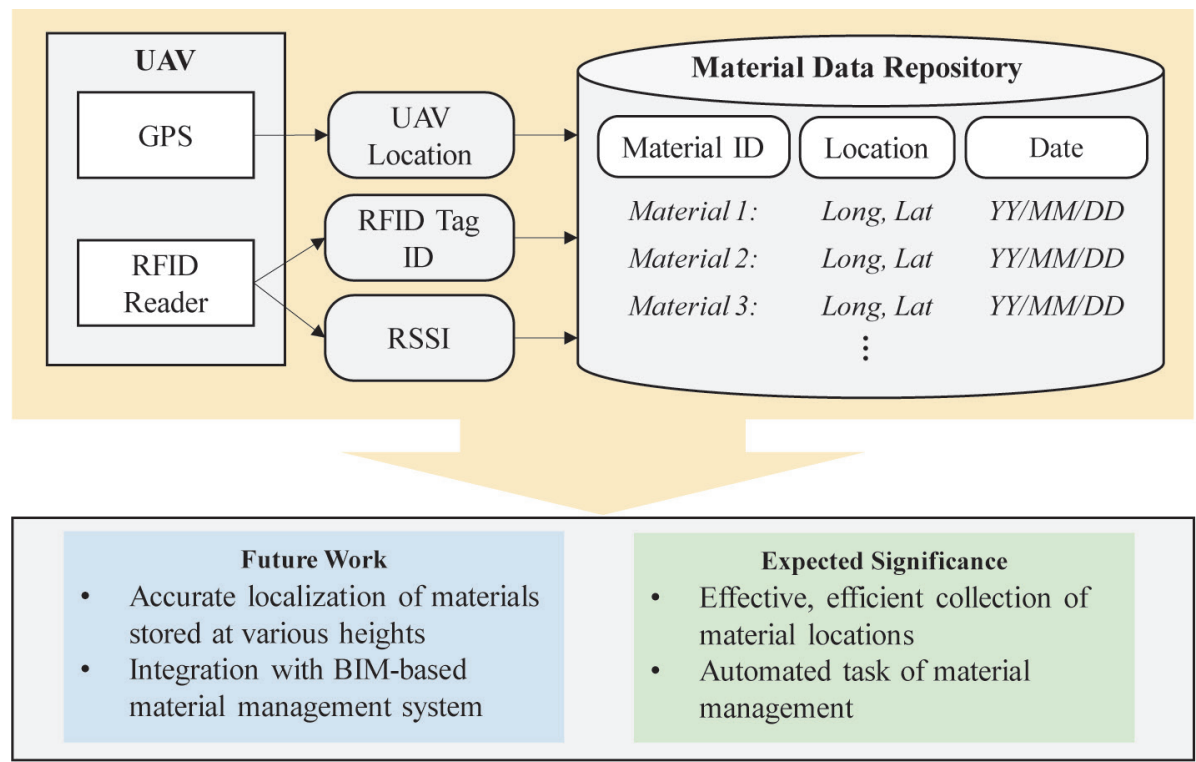

Fig. 2. (Color online) Schematic framework of RFID-based material localization, its impact, and expected future work.

the feasibility of the use of the proposed platform. Further development of the proposed platform regarding RSSI analysis as well as building information modeling (BIM)-based data management and visualization will lead to advanced material management (Fig. 2).

\subsection{UAV-RFID platform: hardware and software}

The UAV model employed in this research is DJI Matrice 100, which is a quadcopter made for developers. ${ }^{(17)}$ It allows developers to install additional sensors beside a GPS, a camera, a gyroscope, and an inertial measurement unit, and to embed newly developed codes for data analysis, wireless communication, and hardware control. In this research, the UAV model is used to collect GPS data and to carry an RFID reader. The specifications of the UAV model are shown in Table 1.

There are two types of RFID tags - active and passive. The active tags are powered by an embedded battery to spread their own signals. ${ }^{(18)}$ When a tag receives a signal from a reader, it sends its own signal back to the reader. Also, beacon types of active tags simply emit their own signals consistently regardless of signal reception, which is appropriate for tolling high-speed vehicles. The active tags exhibit generally long read distances up to around $100 \mathrm{~m}$. However, the use of batteries limits their lifetime to within 3-5 years. Also, they are relatively bulky and their price is high.

On the other hand, the passive tags have no internal power source and rely on the electromagnetic energy transferred from a reader. ${ }^{(18)}$ Accordingly, their price is usually much lower than that of the active tags, which is practically a major advantage. The passive tags are relatively small because they do not require batteries; thus, they can last a lifetime. The passive tags are utilized for various purposes such as access control and stock management. From 
Table 1

Specifications of DJI Matrice 100.

\begin{tabular}{lc}
\hline Maximum speed & $17 \mathrm{~m} / \mathrm{s}$ (with no additional payload and no wind) \\
\hline Operating temperature & $-10-40{ }^{\circ} \mathrm{C}$ \\
\hline Hovering duration & $33 \mathrm{~min}$ (with no additional payload) \\
\cline { 2 - 2 } & $22 \mathrm{~min}$ (with $500 \mathrm{~g}$ additional payload) \\
\hline Allowable additional payload & $840 \mathrm{~g}$ \\
\hline Remote controller operating frequency & $2400-2483 \mathrm{GHz}$ \\
\hline
\end{tabular}

the three main frequency ranges used for the passive tags, the ultrahigh frequency (UHF) that ranges from 865 to $960 \mathrm{MHz}$ allows longer read distances up to around $30 \mathrm{~m}$, while the other frequencies can read tags located within $1 \mathrm{~m}$. Although it is known that RFID technology generally does not work well when a tag is mounted on metal surfaces because of signal reflections, there exist specific RFID tags that alleviate the reflection problems to operate smoothly on metal surfaces. ${ }^{(18,19)}$

Considering the normal environment of construction sites, the UAV is prone to collision accident if it flies to a height of around $1 \mathrm{~m}$. The read distance should be long enough to assure a safe flight control. At the same time, the flight altitude should be constrained to as low as possible because a higher flight will decrease the localization precision. In other words, if the UAV identifies a tag at a high altitude, then the range of the possible tag location that the proposed platform can answer becomes larger. This research regards the altitude of around 10-20 $\mathrm{m}$ as appropriate and focuses on steel materials. Accordingly, the tag and reader detailed in Table 2 are determined to fit the purpose of this research. The type of the selected tag's protocol is Gen2, which is a passive type, and the tag (Fig. 3) is specifically developed for metal materials. ${ }^{(20)}$ The read distance is up to $10-15 \mathrm{~m}$ when the tag communicates with a hand-held reader, which is suitable to the plan of this research. The read distance of the reader is up to $25 \mathrm{~m}$, so $10-15 \mathrm{~m}$ is expected to be optimal when using the selected tags and reader. The reader weighs $240 \mathrm{~g}$, which is far less than the UAV payload $(840 \mathrm{~g})$. It is equipped with a built-in linear antenna that emits signals in a flat radial shape as shown in Fig. 3.

As previously mentioned, the reader is attached to the UAV, pointing downwards (Fig. 4). The RFID data is collected by a notebook on the ground station communicating with the reader via Bluetooth. The software application provided by the manufacturer of the reader is installed on the notebook to facilitate the data collection (Fig. 4). The application collects tag IDs and RSSI data.

\subsection{RFID tag localization}

By using the data collected by the hardware and software described in Sect. 4.2, the locations of the identified tags are approximated as illustrated in Fig. 5. The proposed system gives the tag location as a certain range of a region within which the tag is expected to be placed. The latitude and longitude of the UAV, recorded when a tag is identified, are used as a centroid of the probable region. The scale of the probable region is inversely related to the localization precision. In other words, the precision is higher when the system determines a tag location 
Table 2

Specifications of the employed RFID tag and reader.

\begin{tabular}{cc}
\hline RFID tag & \\
\hline Long distance metal tag & RFID reader $^{(22)}$ \\
Protocol: EPC Class1 Gen2 (Passive) & Model name: Swing U \\
Read distance & RF output: $+30 \mathrm{dBm}$ \\
Fixed reader: $20-25 \mathrm{~m}$ & Read distance: $0-25 \mathrm{~m}$ \\
Hand-held reader: $10-15 \mathrm{~m}$ & Communication: Bluetooth, USB \\
Dimension: $64 \times 55 \times 13 \mathrm{~mm}^{3}$ & Dimension: $260 \times 110 \times 40 \mathrm{~mm}^{3}$ \\
Weight: $43 \mathrm{~g}$ & Weight: $240 \mathrm{~g}$ \\
\hline
\end{tabular}

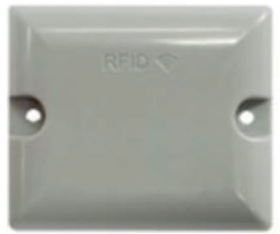

(a)

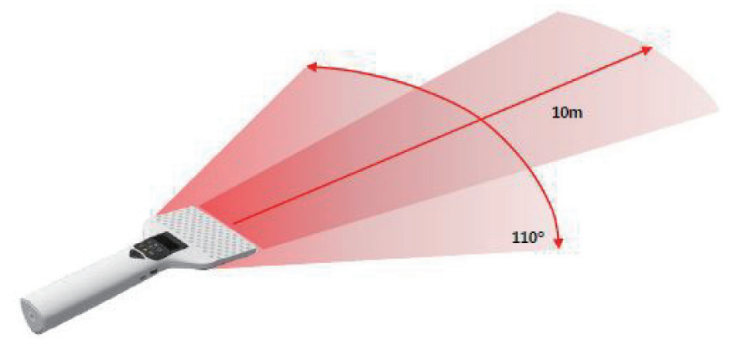

(b)

Fig. 3. (Color online) RFID hardware: (a) tag and (b) hand-held reader. ${ }^{(21,22)}$

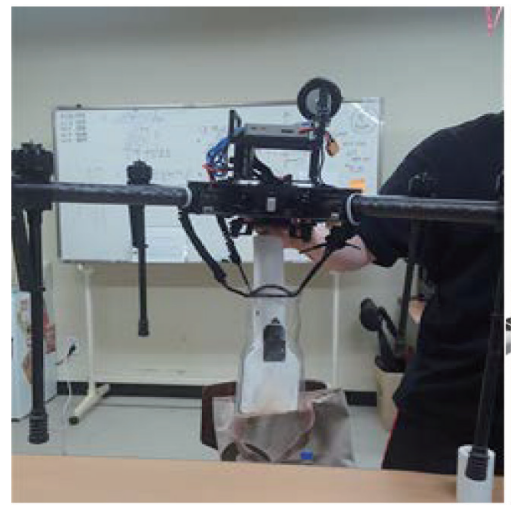

(a)

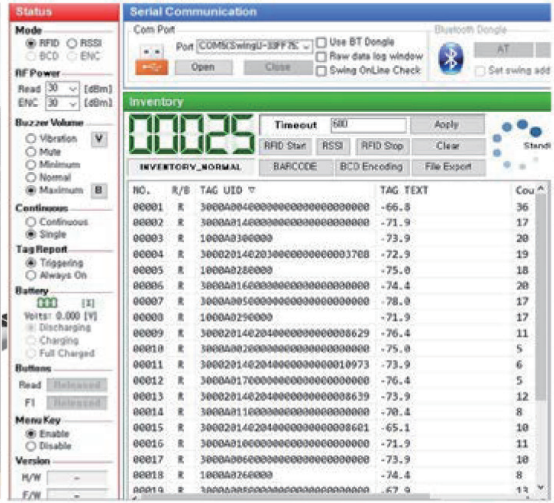

(b)

Fig. 4. (Color online) (a) RFID reader fixed on the UAV and (b) RFID data collection software.
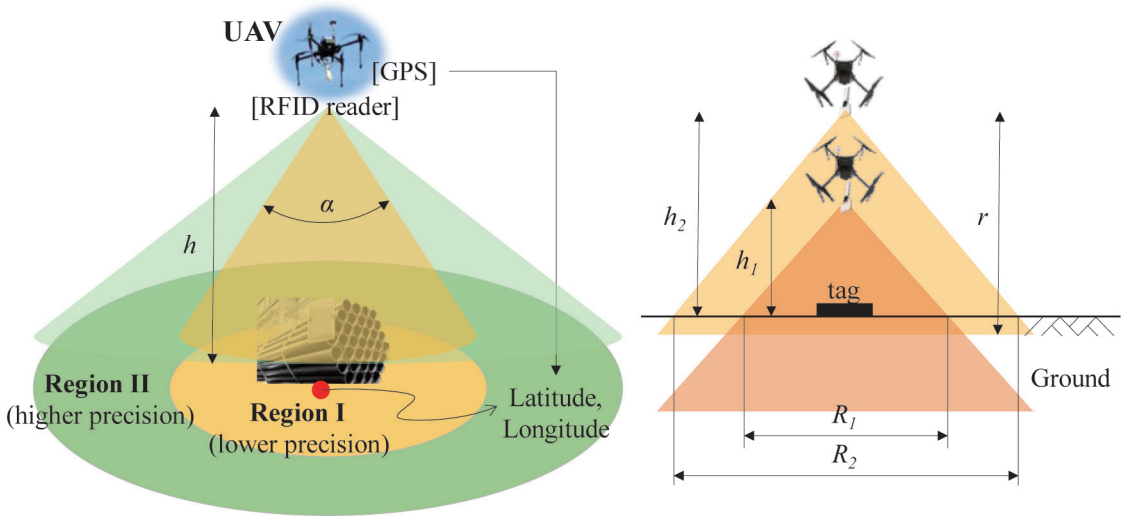

Fig. 5. (Color online) Schematic overview of the tag localization and critical parameters. 
more as a probable region of $1 \times 1 \mathrm{~m}^{2}$ than as a probable region of $10 \times 10 \mathrm{~m}^{2}$. The scale of the probable region is determined depending on the read distance $(r)$ and signal emission angle $(\alpha)$ of the reader as well as the flight altitude $(h)$. When it comes to the precision of the localization, lower altitudes $\left(h_{1}>h_{2}\right)$ are preferred since the probable regions $\left(R_{1}>R_{2}\right)$ are determined to be smaller, leading to higher precision levels. Similarly, narrower angles of the signal emission $(\alpha)$ result in smaller probable regions and accordingly higher precision levels.

From the perspective of tag reading performance, the probable region is equivalent to the sensing range. Note that the flight altitude $(h)$ should be maintained lower than the reader's read distance $(r)$ to secure a sufficient level of tag reading performance. Under this condition, the sensing range increases $\left(R_{1}\right)$ as the UAV flies higher $\left(h_{1}\right)$. However, the signal becomes relatively weaker because the distance from the tag becomes longer. On the other hand, the lower altitude $\left(h_{2}\right)$ would guarantee stronger signals but decrease the chances to have tags within the sensing range $\left(R_{2}\right)$. In other words, regarding the tag reading performance, there is a trade-off between the sensing range and the signal strength depending on the flight altitude.

Accordingly, it is necessary to determine the read distance $(r)$ and signal emission angle $(\alpha)$ of the RFID instruments, and the appropriate flight altitude $(h)$. Section 5 will present a series of experiments conducted to address these issues.

\section{Experiments and Results}

This section consists of four experiments. The first experiment is for determining the read distance $(r)$ and signal emission angle $(\alpha)$. The second and third experiments are for revealing the relationship between the scale of the probable region $(R)$ and the flight altitude. The scale of the region is measured as read ranges in two different orthogonal directions-longitudinal and transverse. Through the three experiments, an optimal altitude $(h)$ is also determined. On the basis of the experimental results, the proposed system is evaluated in the fourth experiment that tests the tag localization performance.

\subsection{Performance of reading RFID tags}

The proposed platform is tested on steel materials to confirm the maximum read distance of the selected tags when attached to metal surfaces. A tag is attached to a steel plate of $200 \times 300 \times 30 \mathrm{~mm}^{3}$, and RSSI data are recorded by varying the distance between the tag and the reader with $1 \mathrm{~m}$ intervals. The experiments in this section are all conducted without attaching the reader to the UAV. From the experiments, it is found that the tags can be identified when they are located within $20 \mathrm{~m}$ from the reader. The experiments are extended to reveal the relationship between the RSSI and the distance from the reader to a tag. At this time, the distance is varied to range from 2 to $20 \mathrm{~m}$ with $2 \mathrm{~m}$ intervals, and the RSSI is recorded three times per tag for each distance. Sixteen tags are tested for every distance, generating a total of 48 RSSI data for each distance. Figure 6 shows the average RSSI values versus the distance. It is observed that the RSSI and distance are inversely proportional to each other. This distinct pattern signifies the potential that the RSSI data can be used to estimate the tag locations if 


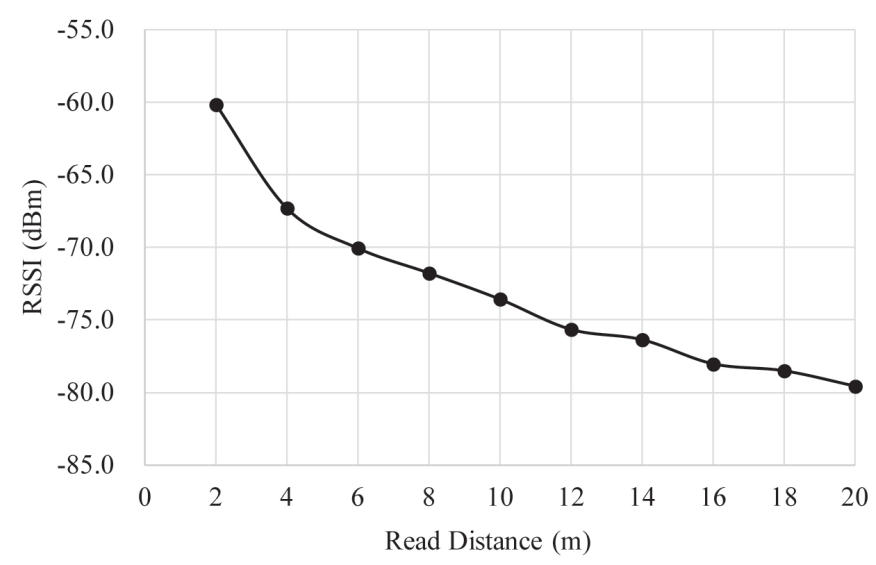

Fig. 6. Relationship between received signal strength and read distance.

the pattern is still visible even when the reader is attached to the UAV. Although the standard deviation for the read distances of 2 and $4 \mathrm{~m}$ is around $1.4 \mathrm{~m}$, that for the read distances larger than $12 \mathrm{~m}$ is around $3.0 \mathrm{~m}$. The standard deviation indicates that the larger the distance, the lower the reliability of the RSSI data.

Since the selected reader has a specific pattern in radiating signals as shown in Fig. 3, an additional experiment is conducted to test the reading performance using the angle between the reader and the tags. Fifteen tags are arranged along an arc of $5 \mathrm{~m}$ radius with $10^{\circ}$ intervals (Fig. 7). The angle of the arc is $140^{\circ}$ from -70 to $70^{\circ}$, and the reader is located at the arc center pointing at the midpoint of the arc $\left(0^{\circ}\right)$. The reader is held horizontally having the flat surface of the head parallel to the ground surface (Fig. 7). RSSIs are recorded three times per tag and their average values are shown in Fig. 8. It is observed that the tags located at both ends of the range are not detected. Also, as expected, the strongest signals are obtained from the tag located along the reader's pointing direction $\left(0^{\circ}\right)$. The received signal strength decreases as the angle between the reader's pointing direction and the tag location increases. This signifies that the reader's pointing direction also acts as an important factor affecting the reading performance. From the results, it is determined that the signals emitted within $\pm 50^{\circ}$ are reliable enough for tag localization.

\subsection{Horizontal read range with varied UAV altitudes}

\subsubsection{Longitudinal read range}

After attaching the reader to the UAV (Fig. 4), experiments are conducted to investigate the effect of the altitude on the reading performance. In the first experiment, the maximum flight altitude at which tags can be read is measured to be $13 \mathrm{~m}$. Then, the second experiment is conducted to determine the horizontal read range along the flight direction (longitudinal read range) in relation to the flight altitude. The longitudinal read range is measured by taking the following steps (Fig. 9). 

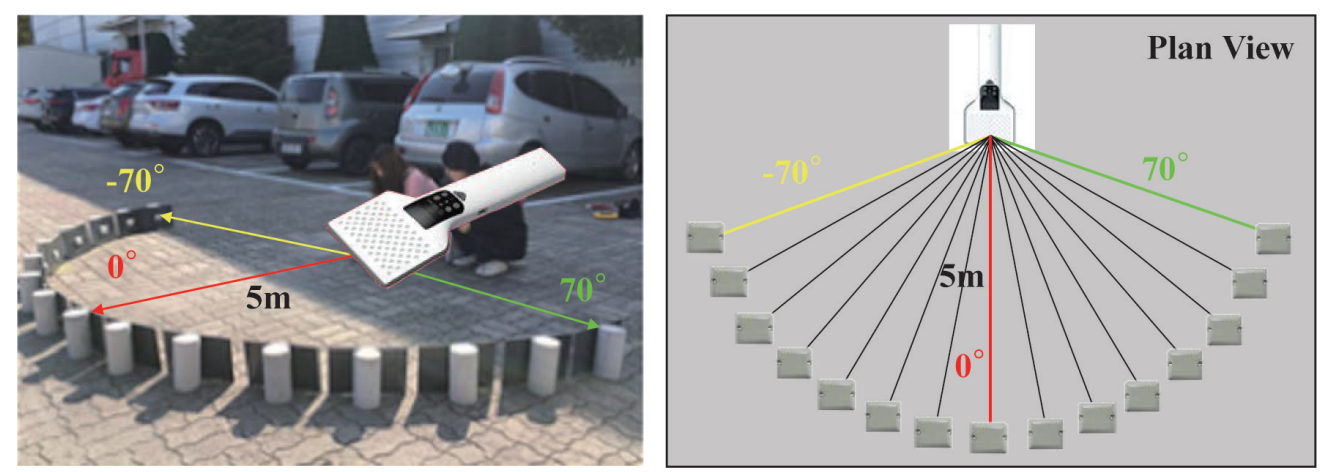

Fig. 7. (Color online) Experimental setup for testing reading performance according to reader's pointing direction.

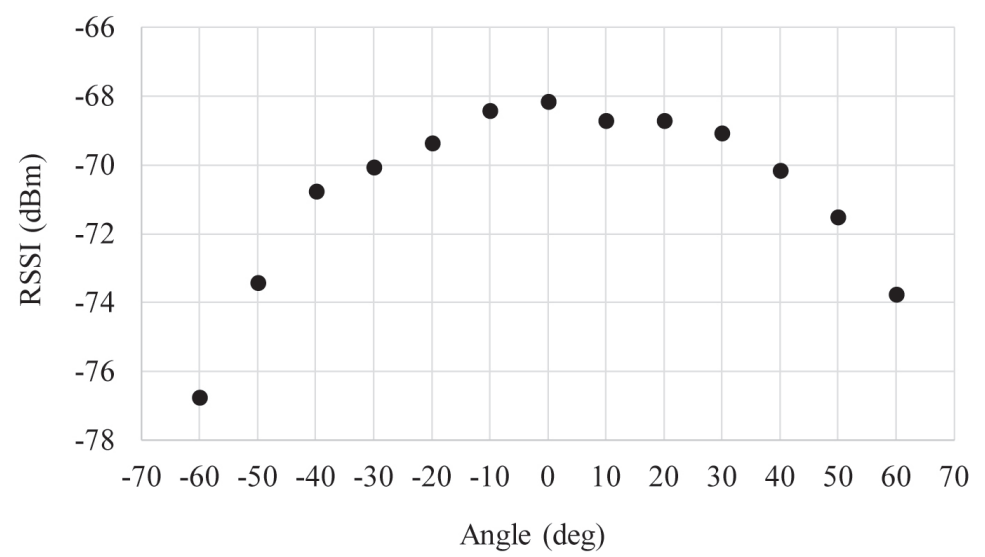

Fig. 8. Relationship between RSSI and reader's pointing direction.
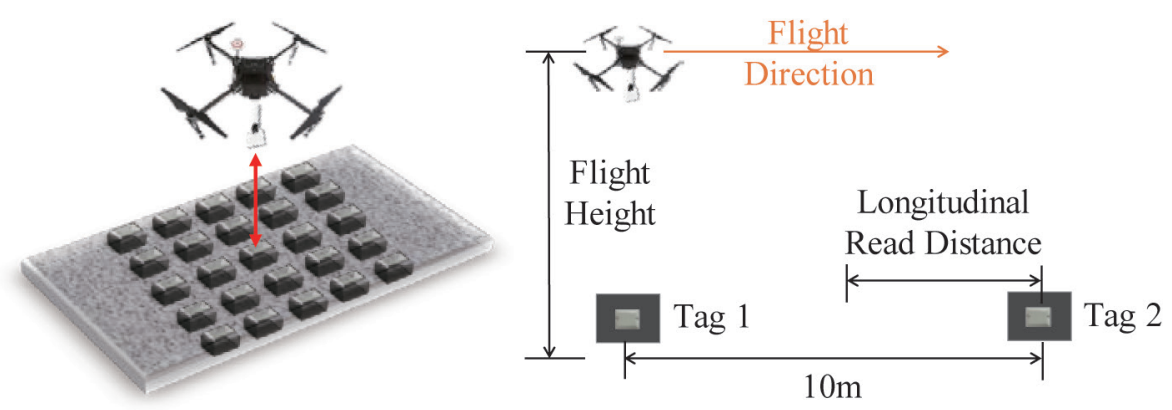

Fig. 9. (Color online) Experimental setup for measuring longitudinal read range.

(1) The UAV is controlled to a position in the vertical direction of Tag 1.

(2) Once the reader identifies the tag below, the UAV is moved toward Tag 2 located 10 m away from Tag 1.

(3) The latitude and longitude of the UAV are recorded when Tag 2 is read.

(4) The longitudinal read distance is measured as the distance between the location recorded in step (3) and the location of Tag 2. 
The horizontal read distances are measured for various altitudes of 3, 6, 9, 12, and $13 \mathrm{~m}$. The result is shown in Fig. 10. As shown in the figure, the longest horizontal read range is recorded as $3.79 \mathrm{~m}$ when the UAV flies at the altitude of $9 \mathrm{~m}$. It is obvious that the range decreases as the altitude increases above $9 \mathrm{~m}$ because of the tags' read range, which is $10-15 \mathrm{~m}$ when being operated with a hand-held reader. In other words, when calculating the actual distance from the reader located $12 \mathrm{~m}$ above a tag, the distance to the tag located horizontally farther than $2.5 \mathrm{~m}$ away would exceed the tag's read range. On the other hand, it is inferred that the longitudinal read range decreases as the altitude decreases because of the reader's reading angle determined in Fig. 8.

\subsubsection{Transverse read range}

Similar to the previous experiment, an experiment is planned to determine the horizontal read range along the transverse direction of the flight and to evaluate the tag reading performance depending on the flight altitude. Fifteen steel plates to which tags are attached are arranged on the ground (Fig. 11). Since this research considers controlling the UAV to be higher than $10 \mathrm{~m}$ to avoid collisions, the experiment is conducted at three altitudes of 11, 12, and $13 \mathrm{~m}$. The dimension of the grid marked as a blue rectangle in Fig. 11 is determined from the

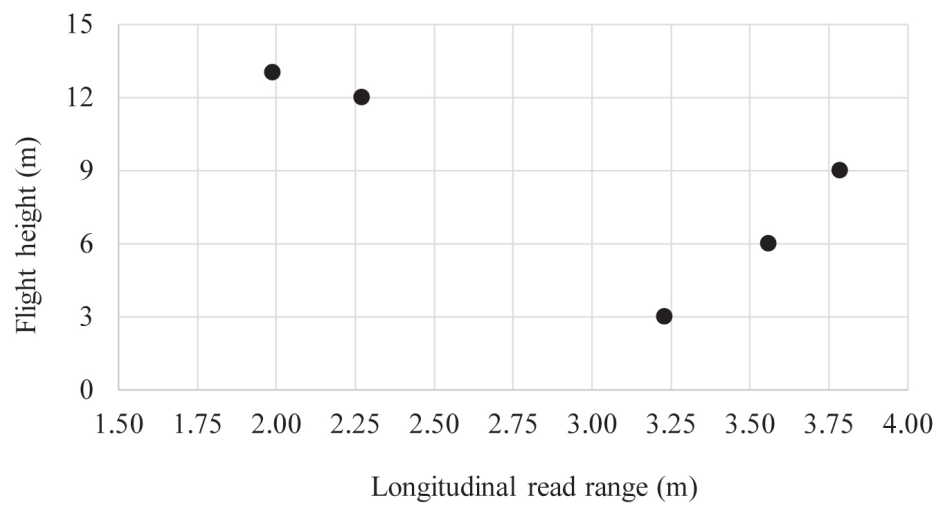

Fig. 10. Longitudinal read range depending on the flight height.
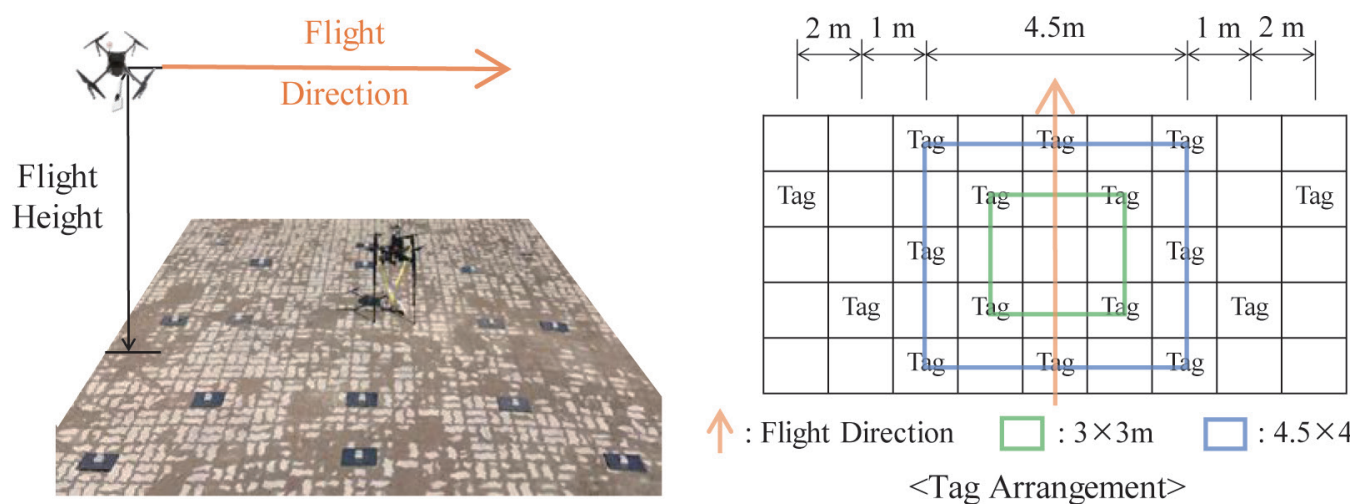

个: Flight Direction $\quad \square: 3 \times 3 \mathrm{~m}$

$: 4.5 \times 4.5 \mathrm{~m}$

$<$ Tag Arrangement $>$

Fig. 11. (Color online) Experimental setup for measuring transverse read range. 
longitudinal read range obtained at the altitude of $12 \mathrm{~m}$ (Fig. 10). In other words, provided that the longitudinal read range of the $12 \mathrm{~m}$ flight altitude is approximately $2.25 \mathrm{~m}$, the dimension of the blue rectangle is determined as twice of it $(4.5 \mathrm{~m})$. In the experiment, how wide the reader's signal can reach is investigated by checking which tags are read by the reader while flying the UAV in the direction of the orange arrow in Fig. 11 at different altitudes. The experiment is repeated four times for each altitude. The results are presented in Fig. 12. When the altitude is $11 \mathrm{~m}$, all tags located within the $6.5 \mathrm{~m}$ width in the transverse direction are identified clearly, while the two tags located beyond the $6.5 \mathrm{~m}$ width are not read at all. On the other hand, when the altitude is 12 or $13 \mathrm{~m}$, the tag reading performance is not consistent regardless of the tag location. Accordingly, $11 \mathrm{~m}$ is determined to be the most appropriate flight altitude that has a distinct transverse read range and exhibits consistent reading performance within the range.

\subsection{Tag localization}

On the basis of previous experiments, the final experiment is performed to test the feasibility of tag localization. The experiment plan is illustrated in Fig. 13. The flight altitude is set as $11 \mathrm{~m}$ since it is determined to be the most appropriate in the previous experiment. Thirty tags are arranged on the ground on the basis of the planned UAV path. Six tags are placed along the path, and fourteen tags are placed along the lines that are shifted from the path by $2.5 \mathrm{~m}$ to each side. In other words, these twenty tags are located within the transverse read range. Ten additional tags are located beyond the transverse read range. The test is repeated eight times, and the number of times each tag is read is shown in Fig. 14. The six tags along the planned path are identified in all eight tests. However, the tags around the green border (Fig. 14) on the right side of the path are read more than expected, while the tags on the other side are read less than expected. The reason for the shifted results is inferred to be attributed to the errors of the flight control and GPS. In several tests out of eight, because of the wind, the UAV was forced to be slightly off the planned path toward the right side as displayed with an orange arrow in Fig.

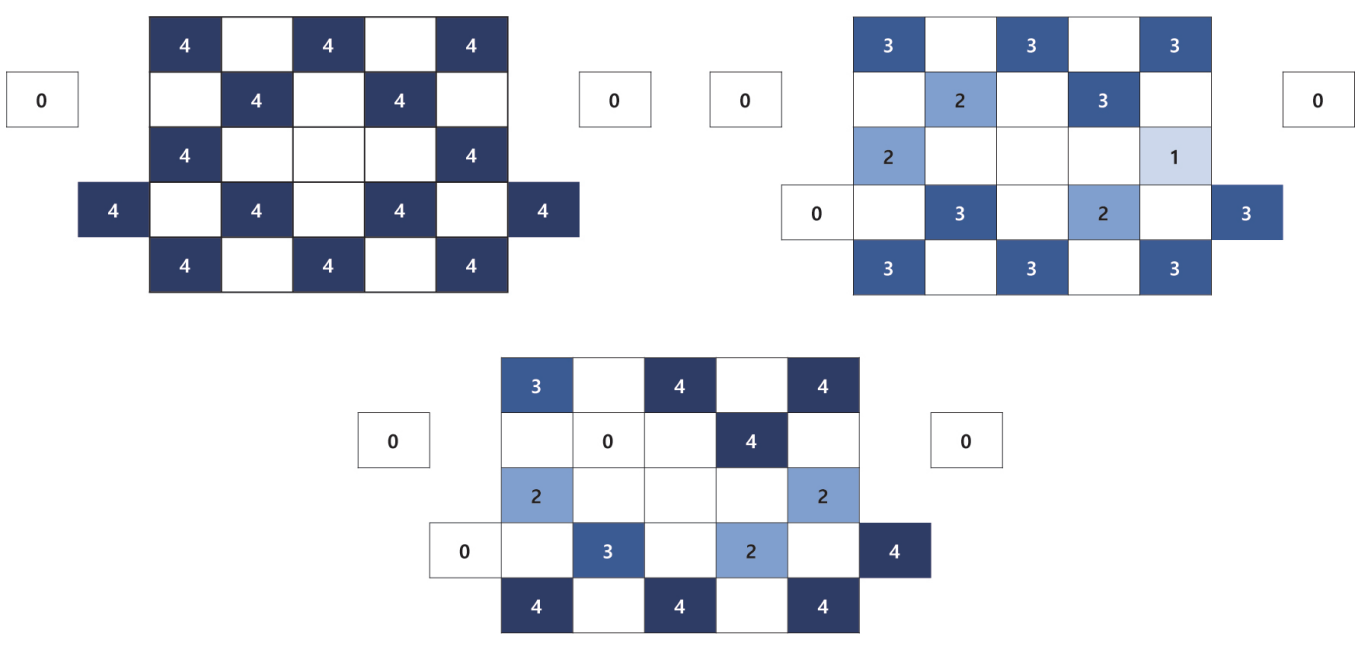

Fig. 12. (Color online) Read count of the tags from four repeated tests. 


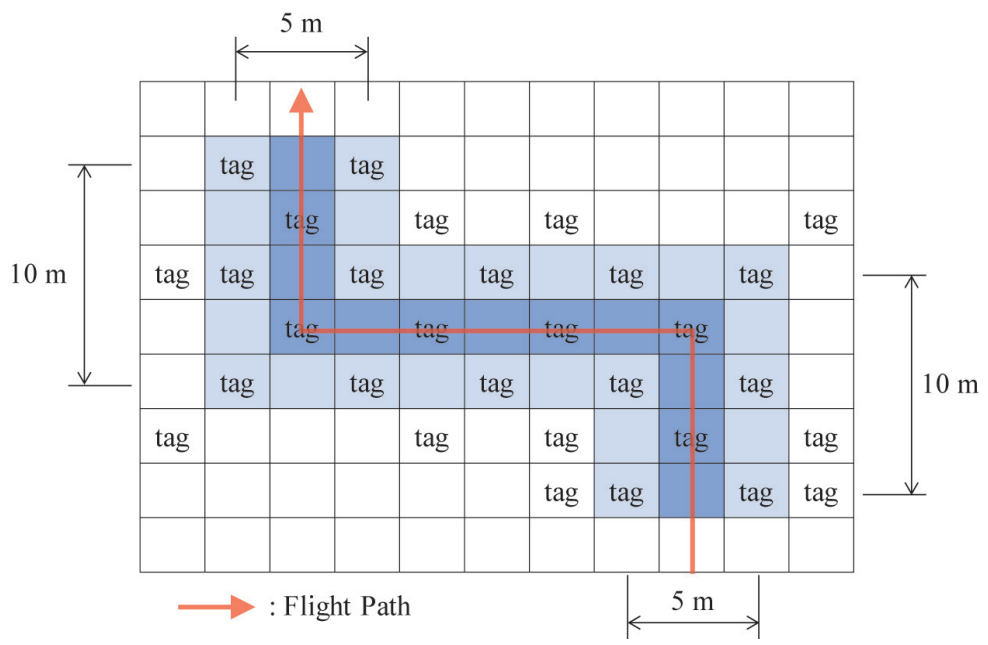

Fig. 13. (Color online) Experimental setup for tag localization.

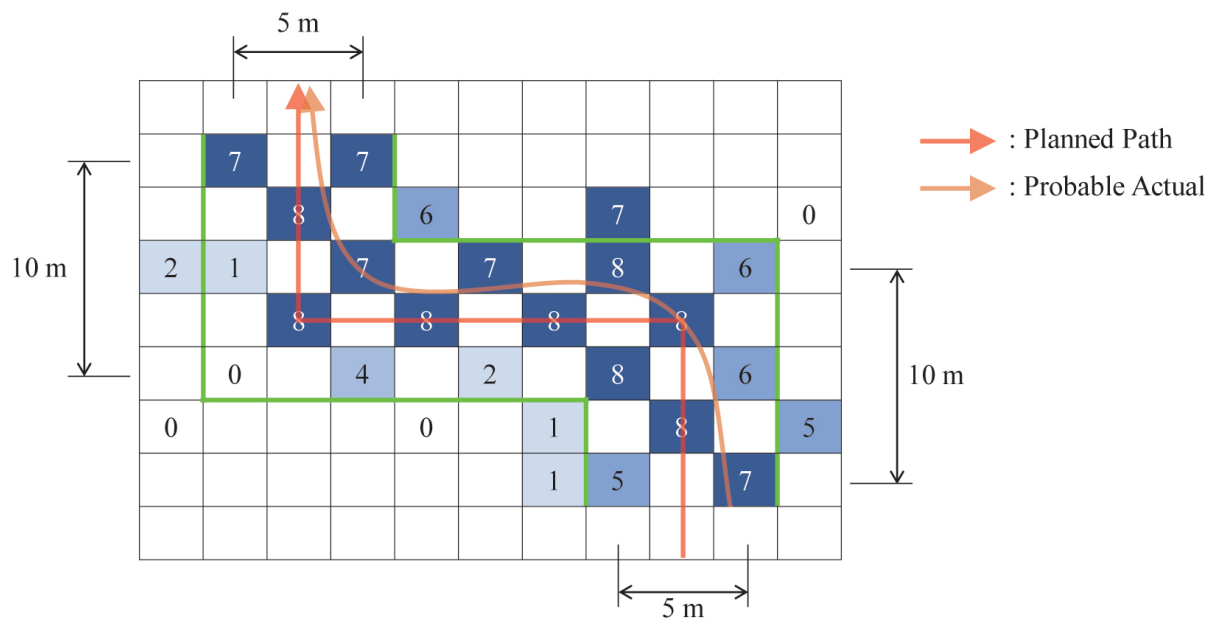

Fig. 14. (Color online) Tag reading results.

14. At the same time, larger errors may be generated in the GPS data. Despite the errors, the results demonstrate a pattern that the tags located within the transverse read range of the UAV flight path are read with a higher possibility. Accordingly, on the basis of the experimental results, the platform can provide probable regions of tag locations in the form of about $5 \times 5 \mathrm{~m}^{2}$ rectangles. This suggests that the proposed platform has a great potential to localize RFID tags on construction resources.

\section{Discussion}

In this paper, we present the important factors to consider when localizing tags using a UAV, as well as the required experiments to determine the effects of the factors on the localization performance and precision. Although the experiments in Sect. 5 show the potential feasibility of the proposed system for material localization on construction sites, the system should 
be enhanced in various ways for practical implementation. First, the proposed system only provides a probable region of a tag with a precision level of a $5 \times 5 \mathrm{~m}^{2}$ rectangular range, instead of providing a single coordinate. For more practical applications, the precision level should be narrowed down to a smaller range or ultimately to a single point. Further advanced analysis of the signal strength and UAV posture data may be required to achieve a higher precision level. Testing various types of RFID sensors and UAVs may also be required to find optimal combinations.

Second, all tags are assumed to be located on a flat ground surface. In other words, the tags are assumed to be at zero altitude. To consider other cases in which tags are located at various altitudes, additional experiments should be conducted.

Third, the results of this study are limited to the cases of using the reader that has a radial shape of the signal emission, and a reading distance range of about 10-20 m. Using other types of readers would result in different outcomes. For improving the precision level of tag localization, the readers with directional antennas would be preferred to omnidirectional or radial ones since the scale of the corresponding probable region can be narrowed down significantly. Also, the directional antennas generally emit stronger signals, resulting in longer read distances. However, the use of the directional antennas may require denser profiles of flight routes to cover all spatial areas with smaller sensing ranges. Practitioners should determine all these factors appropriately on the basis of the project conditions such as the size of the site and the preferred level of localization precision.

Fourth, the hardware setup can be upgraded for better performance. For example, during the presented experiments, the reader is not installed firmly enough to avoid its swinging motions, which may create inconsistent signal communications. In practical implementation, more advanced mechanical setups such as a gimbal will be required to have the signal direction pointing downward consistently. Certain types of devices to avoid signal interferences will also be helpful. For example, if the reader can be stretched out lower than the landing legs, the effect of the reflections from the UAV frames will decrease. Furthermore, the use of a real-time kinematic GPS (RTK-GPS) will be helpful to increase the accuracy of the localization.

Lastly, preparing a guideline of the UAV flight control will allow achieving consistent performance regarding both tag reading and localization. For example, since the UAV's movements also directly affect the antenna's direction, the flight should be constrained to a lower speed especially when making a turn. In addition, the flight should be planned efficiently to reduce battery consumption, and conversely, enough batteries should be prepared to cover the planned flights. The flights should be scheduled on the basis of the weather and the batteries' allowable operating temperatures. Also, the flight plan should reflect the fact that batteries are consumed more rapidly at low temperatures.

\section{Conclusions}

For effective material management on construction sites, it is critical to monitor the material locations consistently. However, in a complex crowded or large-scale open site, it is hardly possible to track all materials without the help of sensing technologies. In this perspective, 
various technologies including RFID, GPS, and UWB technologies have been investigated continuously. As a result of continuous research effort, RFID technology is introduced in real projects to track important resource materials such as precast concrete elements and large-scale steel pipes. Although the technology enhances the efficiency and quality of the material management tasks, the following limitations that should be overcome still remain: the requirement of manual effort to carry hand-held readers over the sites and very limited available spots of data collection when using fixed readers. To resolve these issues, a new platform that fuses the UAV and RFID technologies is proposed in this study. In this study, we focused on localizing steel materials with a UAV flying over $10 \mathrm{~m}$ or higher. The hardware and software of the UAV are examined in detail and the basic strategy to localize tags is described thoroughly. Several stages of experiments are conducted to determine the detailed specifications and performance of the employed hardware, and finally the feasibility of tag localization is tested on the basis of the precedented experimental results. The results show that the proposed platform can be used to localize tags with a precision scale of $5 \times 5 \mathrm{~m}^{2}$ rectangular regions. This research is expected to be extended to further enhance the precision and enable the pin-pointing of the tag locations through the in-depth analysis of the RSSI data and UAV motions.

\section{Acknowledgments}

This work was supported by the Basic Science Research Program through the Ministry of Science and ICT and the National Research Foundation of Korea (NRF-2016R1C1B2014997).

\section{References}

1 W. Lu, G. Q. Huang, and H. Li: Autom. Constr. 20 (2011) 101.

2 E. J. Jaselskis, M. R. Anderson, C. T. Jahren, Y. Rodriguez, and S. Njos: J. Constr. Eng. Manag. 121 (1995) 189.

3 B. Akinci, M. Pattin, and E. Ergen: Int. Symp. Autom. Rob. Constr. 19th ISARC (2002) 381.

4 E. J. Jaselskis and T. El-Misalami: J. Constr. Eng. Manag. 129 (2003) 680.

5 P. M. Goodrum, M. A. McLaren, and A. Durfee: Autom. Constr. 15 (2006) 292.

6 E. Ergen, B. Akinci, and R. Sacks: Autom. Constr. 16 (2007) 354.

7 S. Y. L. Yin, H. P. Tserng, J. C. Wang, and S. C. Tsai: Autom. Constr. 18 (2009) 677.

8 E. Valero and A. Adan: Measurement 94 (2016) 614.

9 S. Rathinam, Z. W. Kim, and R. Sengupta: J. Infrastruct. Syst. 14 (2008) 52.

10 C. Zhang and A. Elaksher: Comput.-Aided Civ. Infrastruct. Eng. 27 (2011) 118.

11 J. Irizarry, M. Gheisari, and B. N. Walker: J. Inform. Technol. Constr. 17 (2012) 194.

12 S. Siebert and J. Teizer: Autom. Constr. 41 (2014) 1.

13 R. Ashour, T. Taha, F. Mohamed, E. Hableel, Y. A. Kheil, M. Elsalamouny, M. Kadadha, K. Rangan, J. Dias, L. Seneviratne, and G. Cai: 2016 IEEE 59th Int. Midwest Symp. Circuits and Systems (MWSCAS) 2016.

14 N. Metni and T. Hamel: Autom. Constr. 17 (2007) 3.

15 G. Morgenthal and N. Hallermann: Adv. Struct. Eng. 17 (2014) 289.

16 S. Bang, H. Kim, and H. Kim: Autom. Constr. 84 (2017) 70.

17 DJI, Matrice 100, https://www.dji.com/kr/matrice100 (accessed September 1, 2018).

18 S. Smiley: https://blog.atlasrfidstore.com/active-rfid-vs-passive-rfid (accessed September 1, 2018).

19 J. Thrasher: https://blog.atlasrfidstore.com/rfid-tags-on-metal-surfaces (accessed September 1, 2018).

20 SkyRFID Inc.: RFID Gen 2 - What is it? - Smart RFID, http://skyrfid.com/RFID_Gen_2_What_is_it.php, (accessed September 1, 2018).

21 BONANETWORKS: Specific Tag-900 MHz, http://www.bonanetworks.com/index-3.html (accessed September 1, 2018). 
22 NETHOM (2014) Swing-U Manual: https://www.nethom.co.kr/bbs/bbs download.php?bbs data=aW4PTEzM CZzdGFydFBhZ2U9MCZsaXN0Tm89MiZ0YWJsZT1jc19iYnNfZGF0YSZjb2R1PW5vdGljZSZzZWFyY2hfa XRlbT0mc2VhcmNoX29yZGVyPQ==\%7C\%7C\&download=1 (accessed September 1, 2018).

\section{About the Authors}

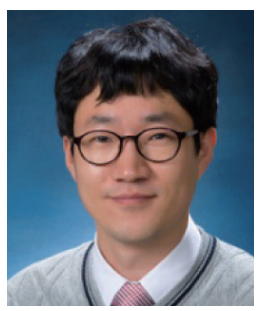

Sanghyeok Kang received his B.S., M.S., and Ph.D. degrees from Hanyang University, Seoul, South Korea in 2003, 2005, and 2009, respectively. From 2009 to 2012, he was an associate specialist at the Safe Transportation Research \& Education Center, University of California, Berkeley, U.S. From 2012 to 2015, he was a research fellow at the Construction Economy Research Institute of Korea. Since 2015, he has been an assistant professor at Incheon National University in South Korea. His research interests include construction automation, construction data analysis, and construction policy.

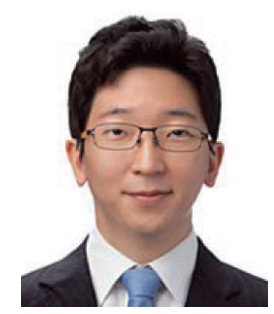

Man-Woo Park received his B.S. and M.S. degrees from Seoul National University, Seoul, South Korea in 2001 and 2003, respectively, and his Ph.D. degree from Georgia Institute of Technology, USA in 2012. From 2013 to 2016, he was an assistant professor at Myongji University in South Korea. Since 2017, he has been an associate professor at Myongji University. His research interests include construction information technology and various sensing technologies for on-site construction monitoring including computer vision and image processing technologies.

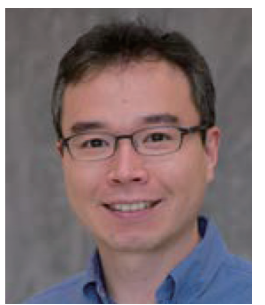

Wonho Suh received his B.S. degree from Seoul National University, Seoul, South Korea in 1998 and his M.S. and Ph.D. degrees from Georgia Institute of Technology, USA in 2008 and 2012, respectively. From 2011 to 2013, he was a research faculty/research engineer at Georgia Institute of Technology, USA. Since 2013, he has been an assistant professor at Hanyang University in South Korea. His research interests include traffic simulation, data analysis, and ad hoc distributed simulation. 